\title{
Cranial nerve involvement in COVID-19
}

Ahmet Doblan ${ }^{1}$, mehmet erkan kaplama ${ }^{1}$, SEMİH AK ${ }^{2}$, Nazli Basmaci ${ }^{3}$, ŞENIZ GÖKTAŞ ${ }^{4}$, SÜLEYMAN GÜLER ${ }^{4}$, EMINE TARINI ${ }^{4}$, and togay müderris ${ }^{5}$

${ }^{1}$ Health Sciences university of turkey, Sanliurfa M. Akif Inan Education and Research Hospital, Department of Otorhinolaryngology, Sanliurfa , Turkey ${ }^{2}$ BALIKLIGÖL STATE HOSPITAL

${ }^{3}$ Health Sciences university of turkey, Sanliurfa M. Akif Inan Education and Research Hospital, Sanliurfa , Turkey

${ }^{4}$ Health Sciences university of turkey, Sanliurfa M. Akif Inan Education and Research Hospital, Sanliurfa , Turkey

${ }^{5}$ Bakircay Universitesi

March 13, 2021

\begin{abstract}
Abstract: Introduction: The COVID-19 disease emerged in Wuhan province of China in November 2019 and spread across the world in a short time, resulting in a pandemic. The first case in Turkey was detected on March 11, 2020. The aim of the current study was to reveal the effects of COVID-19 on cranial nerves by monitoring people infected with the disease based on repeated examinations and surveys. Material and Method: The data of 356 patients with a positive COVID-19 polymerase chain reaction (PCR) test who received treatment between June 2020 and August 2020 in our hospital were prospectively evaluated after the study was approved by the relevant ethics committee. Results: Of the 356 patients included in the study, 47 under the age of 18 years were excluded due to their unreliable examination and anamnesis findings. In addition, seven patients that died while in hospital were excluded from the study due to the lack of examination and survey records during their hospitalization. The data of the remaining 302 patients were statistically analyzed. Symptoms of cranial nerve involvement were observed in 135 patients. Conclusion: The COVID-19 disease caused by the SARS-CoV2 virus commonly results in cranial nerve symptoms. The fact that these findings are more common and severe in COVID-19 than previous SARS and MERS outbreaks suggests that it has a more neurotrophic and more aggressive neuroinvasion. While the negative effects of the virus on sensory functions resulting from cranial nerve involvement are evident, motor functions are rarely affected.
\end{abstract}

\section{COVER PAGE}

TITLE: Cranial nerve involvement in COVID-19

AUTHORS:

1. Ahmet DOBLAN, SBÜ Mehmet Akif Inan Training and Research Hospital,Otorhinolaryngology, Sanlıurfa, Turkey. Ass.Prof. MD. Orcid :0000-0002-7783-8157

2. Mehmet Erkan Kaplama, SBÜ Mehmet Akif Inan Training and Research Hospital, Otorhinolaryngology, Sanlıurfa, Turkey. Ass.Prof. MD. Orcid: 0000-0003-4662-409X

3. Semih Ak, SBÜ Mehmet Akif Inan Training and Research Hospital,Otorhinolaryngology , Sanlıurfa, Turkey. MD. Orcid: 0000-0001-6919-9423

4. Nazlı Basmacı, SBÜ Mehmet Akif Inan Training and Research Hospital,Anesthesiology and Reanimation, Sanlıurfa, Turkey. MD.Orcid: 0000-0002-7715-5073 
5. Emine Zeynep Tarini, SBÜ Mehmet Akif Inan Training and Research Hospital, , Sanlıurfa, Pathology , Sanlıurfa, Turkey. Ass.Prof. MD. Orcıd : 0000-0001-9874-5104

6. Süleyman Güler, SBÜ Mehmet Akif Inan Training and Research Hospital,Neurology, Sanlıurfa, Turkey. MD. Orcid: 0000-0001-7931-7649

7. Şeniz Engür Göktaş, SBÜ Mehmet Akif Inan Training and Research Hospital, Ophthalmology , Sanlıurfa, Turkey. Ass.Prof. MD. Orcıd : 0000-0001-7298-4916

8. Togay Müderris, SBÜ Bozyaka Training and Research Hospital,Otorhinolaryngology, Izmir, Turkey. Prof. MD. Orcid:0000-0003-4014-8176

*The authors declare no conflict of interest.

**The authors declare that they did not receive any financial support for the study.

Corresponding author : Ahmet DOBLAN. Member Faculty, MD. ENT-HNS specialist.

E-MAİL: ahmetdoblan@yahoo.com

TEL: $(+90) 5059485563$

ADDRESS: SBÜ Mehmet Akif İnan Training and Research Hospital, Şanluurfa,

Turkey

Merkez Mahallesi, Esentepe Mah, Ertuğrul Cad, 63300

Haliliye/Şanlıurfa

\section{Cranial nerve involvement in COVID-19}

Abstract:

Aim : The COVID-19 disease emerged in Wuhan province of China in November 2019 and spread across the world in a short time, resulting in a pandemic. The first case in Turkey was detected on March 11, 2020. The aim of the current study was to reveal the effects of COVID-19 on cranial nerves by monitoring people infected with the disease based on repeated examinations and surveys.

Material and Method: The data of 356 patients with a positive COVID-19 polymerase chain reaction (PCR) test who received treatment between June 2020 and August 2020 in our hospital were prospectively evaluated after the study was approved by the relevant ethics committee.

Results: Of the 356 patients included in the study, 47 under the age of 18 years were excluded due to their unreliable examination and anamnesis findings. In addition, seven patients that died while in hospital were excluded from the study due to the lack of examination and survey records during their hospitalization. The data of the remaining 302 patients were statistically analyzed. Symptoms of cranial nerve involvement were observed in 135 patients.

Conclusion: The COVID-19 disease caused by the SARS-CoV2 virus commonly results in cranial nerve symptoms. The fact that these findings are more common and severe in COVID-19 than previous SARS and MERS outbreaks suggests that it has a more neurotrophic and more aggressive neuroinvasion. While the negative effects of the virus on sensory functions resulting from cranial nerve involvement are evident, motor functions are rarely affected.

Keywords: Covid-19, SARS-CoV2, Cranial Nerves, Neuroinvasion, Neurotropism

\section{What's already known about this topic?}

covid 19 affects the peripheral and central nervous system.

covid 19 also causes cranial nerve involvement.

What does this article add? 
We have revealed the frequency of cranial nerve involvement in people infected with covid 19.

We also demonstrated this involvement in patients without complaints.

Review criteria: how did you gather, select and analyze the information you considered in your review?

All patients were followed up by the researchers at the time of hospitalization and during the hospitalization period, and their data were collected.

They were also followed by phone calls and questionnaires after discharge.

Message for the clinic: what is the 'take-home' message for the clinician?

covid 19 virus causes very frequent cranial nerve damage.

mostly only covid-19 treatment is sufficient for the regression of symptoms.

some symptoms may last longer than 3 months.

Close follow-up of patients with cranial nerve involvement is important, as there are no long-term covid 19 follow-up results.

Introduction:

The COVID-19 disease emerged in Wuhan province of China in November 2019 and spread across the world in a short time, resulting in a pandemic (1). The first case in Turkey was detected on March 11, 2020. Since then, the disease has continued to spread rapidly, and the number of cases has increased in all provinces. Coronaviruses are a large family of viruses that are seen as the causative agent of a wide range of clinical symptoms from the common cold to severe pneumonia, such as Middle East Respiratory Syndrome (MERS$\mathrm{CoV}$ ) and Severe Acute Respiratory Syndrome (SARS-CoV). SARS-CoV2, responsible for the COVID-19 disease, is considered to be the most effective of this family of viruses, which has led to the ongoing pandemic (2). Disease symptoms may vary depending on the patient's additional diseases and state of the immune system (3). In previous studies, it was shown that most of the symptomatic cases affected by this disease had underlying comorbidities, such as diabetes, hypertension, and heart diseases (4). However, studies conducted to date have reported different rates for these comorbidities.

The incubation period of COVID-19 is around five days in the initial stage of the disease, and although the most common complaints are fever, cough, myalgia, headache, and joint pain, patients can also present with many different symptoms, including taste and smell disorders, diarrhea, and weakness. Complaints due to the involvement of different cranial nerves are also observed in a significant number of patients. Determining the distribution of these carnal nerve impairments according to age, gender and complaints, and revealing the prevalence in infected people can provide guidance in the combat against the disease.

In this context, we aimed to reveal the frequency and severity of cranial nerve symptoms in COVID-19positive patients that presented to a pandemic hospital located in the Southeast Anatolia region in Turkey.

Material and Method:

After receiving the ethics committee approval of Harran University (dated 15.06.2020 and numbered $\mathrm{HRU} / 20.11 .16$ ), the data of 356 patients with a positive polymerase chain reaction (PCR) test for COVID-19, who received treatment in our hospital between June 2020 and August 2020 were prospectively evaluated. The only inclusion criterion was accepted as PCR test positivity. Patients without a PCR test result or those with a negative test result despite the presence of clinical and radiological signs of COVID-19 were excluded from the study. The patients were divided into two groups according to their clinical conditions: those that presented to the hospital with complaints and had a positive PCR result, and those that did not have any related complaint but were detected to have a positive PCR result during routine screenings. In our hospital, all nasopharyngeal swab samples have been being taken by otorhinolaryngologists since 
the onset of the pandemic. In addition, all physicians are assigned to work in hospital services and intensive care follow-up. This allowed our study team to carry out the entire treatment and evaluation process from the patients' examination at the time of presentation to discharge. All demographic characteristics of the patients, namely age, gender, height, and weight, as well as presentation complaints, examination findings, and hematological and radiological data were evaluated. The patients were questioned in terms of comorbidities (hypertension, heart diseases, diabetes mellitus, asthma, and chronic obstructive pulmonary disease). Medical treatment and maintenance treatments received by the patients during hospitalization and after discharge were recorded. The duration of the patients' stay in hospital services and the intensive care unit, as well as the recovery time of their symptoms were also evaluated by our study team. Asymptomatic patients that were incidentally diagnosed with COVID-19 by screening were only followed up. Symptoms that emerged during the follow-up were evaluated and noted.

If the patients were PCR positive, they were routinely treated in the hospital regardless of their complaints. During the treatment, all cranial nerve examinations were performed by otorhinolaryngologists, neurologists and ophthalmologists on the first day of hospitalization and on the day of discharge. In order to increase compliance with follow-up after discharge and to evaluate changes in complaints after discharge, a survey form consisting of yes/no 19 questions was administered to all patients during and after hospitalization (Fig. 1). At the end of treatment, when a swab was taken for the pre-discharge control PCR test, the patient was examined again, and the same survey was used to evaluate the regression of complaints or the emergence of new pathologies.. All patients who were planned to be discharged at the end of the treatment were discharged after the last cranial nerve evaluations by otorhinolaryngologists, neurologists and ophthalmologists. The survey was repeated on the $15^{\text {th }}$ and $30^{\text {th }}$ days after discharge by telephone communication. The questions in this survey inquired whether there was a change in the already known clinical condition of the patient or whether a new complaint appeared. In case of a newly developed pathology, the patient was called back to the hospital for a re-examination. For the patients without any newly developed pathological condition, the results of the survey were compared in relation to the symptoms of the cranial nerves at the time of first admission.

In all PCR-positive COVID-19 cases, the otorhinolaryngologists, neurologists and ophthalmologists performed the individual examination of the following nerves: olfactory (sense of smell), optic (visual field, visual acuity, and pupil reflex), oculomotor (eye movement, pupil reflex, and upper eyelid drooping), N. trochlearis (inward gaze restriction), N. trigeminus (corneal reflex, facial hypoesthesia, nasal mucosal hypoesthesia, weakness in masticatory muscles, and asymmetry or weakness in jaw opening), N. abducens (lateral gaze restriction), N. facialis (facial asymmetry, loss of taste, Schimmer's test, corneal reflex, and reduced salivation), (soft palate palsy, gag reflex, swallowing functions, and dysphagia), N. vagus (laryngeal examination, vocal cord movements, oculocardiac reflex, swallowing functions, pharyngeal reflex, and hoarseness), N. accessorius (weakness in abduction above 90 degrees of the arm, weakness in the sternocleidomastoid muscle, and problems with the rotation of the head), and N. hypoglossus (tongue movement disorder or weakness).

Since there was no excessive accumulation in our hospital due to the pandemic being in the early stages, all cases with a positive PCR test were hospitalized as per our health policy. For this reason, the complaints that developed or regressed during both treatment and hospitalization were closely monitored by examining each patient and communicating with them personally.

Statistical Analysis

Data analysis was performed using IBM SPSS Statistics version 17.0 (IBM Corporation, Armonk, NY, USA). Whether the distribution of continuous variables was normal was determined using the Kolmogorov-Smirnov test. Descriptive statistics for continuous variables were expressed as mean $\pm \mathrm{SD}$ or median (min-max), where appropriate. Numbers and percentages were used for categorical data. While the mean differences between the groups were determined using Student's t-test, the Mann-Whitney U test was applied for the comparison of non-normally distributed variables. Pearson's $\chi^{2}$ test was used in the analysis of categorical data unless otherwise stated. In all $2 \times 2$ contingency tables, to compare categorical variables, the continuity-corrected 
$\chi^{2}$ test was used when one or more of the cells had an expected frequency of 5-25 while Fisher's exact test was used when one or more of the cells had an expected frequency of 5 or less. Multiple logistic regression analyses were performed to determine the best predictor of cranial nerve involvement after the adjustment of data for age and gender. Odds ratios, $95 \%$ confidence intervals, and Wald statistics for each independent variable were also calculated. A p value of less than 0.05 was considered as statistically significant.

Results:

Of the 356 patients included in the study, 47 below 18 years were excluded due to unreliable examination and anamnesis findings. In addition, seven patients who died in hospital were also excluded from the study because of the lack of long-term results. The data of the remaining 302 patients were statistically evaluated.

The cranial nerve involvement of the cases included in the study and their incidence are shown in Table 1. The demographic and clinical characteristics of the cases with and without cranial nerve involvement are summarized in Table 2. There was no significant difference between the groups with and without cranial nerve involvement in terms of age, female-male distribution, body mass index, comorbidities, intensive care requirement, median length of stay in intensive care unit, and median length of hospital stay ( $\mathrm{p}>0.05)$. However, the rate of the patients diagnosed upon complaint was significantly higher in the group with cranial nerve involvement compared to the group without involvement, while the rate of those diagnosed by screening was significantly lower $(\mathrm{p}<0.001)$ (Figure 2$)$.

The frequency distribution of the cases in terms of presentation complaints is shown in Table 3. No significant difference was observed between the groups in terms of cough, diarrhea, respiratory distress, and hoarseness ( $p>0.05)$. However, the rates of patients presenting with fever, sore throat, tiredness, headache, and joint pain complaints were statistically significantly higher in the group with cranial nerve involvement compared to the group without this involvement $(\mathrm{p}<0.05)$.

The probability of having cranial nerve involvement was statistically 7.714 times (95\% CI: 4.309-13.811) higher in patients diagnosed upon complaint than those diagnosed by screening $(\mathrm{p}<0.001)$ (Table 4$)$. The most determinant complaint in distinguishing between the cases with and without cranial involvement was headache, followed by sore throat and joint pain $(\mathrm{p}<0.001, \mathrm{p}=0.003$ and $\mathrm{p}=0.016$, respectively). According to the age- and gender-adjusted data, the probability of cranial nerve involvement was 4.062 times (95\% CI: 1.904-8.667) greater for the patients presenting with the headache complaint, 2.357 times greater for those with a sore throat (95\% CI: 1.337-4.155), and 4.216 times greater for those with joint pain (95\% CI: 1.305-13.623) (Table 4).

In 135 of the 302 patients, symptoms of various cranial nerves were detected over the 40-day period from the time of diagnosis. Although many patients had more than one symptom associated with nerve involvement, isolated involvement was also detected, albeit rarely. While most symptoms, such as loss of vision, sudden hearing loss, vertigo, tinnitus, facial paresthesia, and trigeminal neuralgia rapidly regressed with the initiation of the treatment, it was determined that smell problems were the last symptom to be relieved (3-60 days). The latest emerging cranial nerve findings were related to the trigeminal nerve (facial hypoesthesia and weakness in chewing muscles). In all seven patients with the complaints of the trigeminal nerve, the symptoms began to emerge after discharge from hospital (7-13 days). The most resistant nerve to the virus, with no symptoms, was N. hypoglossus.

Sudden loss of vision developed in one patient during the follow-up. Pathological contrast enhancement and edema were detected in bilateral optic nerves in the MRI of the patient. Diplopia, decreased visual acuity, and eye movement disorder were the other eye symptoms seen among the patients. Vestibulocochlear nerve involvement was also very common. Dizziness and tinnitus were observed frequently, and sudden hearing loss was seen in two patients.

The main problem in all but three patients with facial nerve symptoms was the loss of the sense of taste. In the remaining three patients, vague facial paresis findings not exceeding grade 3 were detected.

After discharge from hospital, the patients were called and questioned about their complaints, and 37 patients 
with complaints that lasted more than one month after discharge were called back to the hospital for reexamination. In one patient, there was no reduction in the severity of smell loss that was present at the time of presentation, and this symptom persisted for more than 40 days. A nasal mucosa biopsy was taken from this patient, and oral and nasal steroids were prescribed. Moderate epithelial damage was observed in the biopsy (Figures 3 and 4).

Among the 37 patients with symptoms lasting for more than one month, reduced visual acuity was seen in one patient, tinnitus in four, numbness of the tongue and loss of taste in 12, hoarseness in one, and loss of smell in 19.

Discussion:

There are many studies showing that COVID-19 infection is neurotrophic and neuroinvasive (5-10). The most common neurological symptoms in COVID-19 are encephalopathy, acute cerebrovascular diseases, and acute polyradiculopathy or neuropathies $(6,11)$. Neurological symptoms may occur as direct effects of SARSCoV-2 virus neurotropism on central and peripheral nervous systems (CNS and PNS), or as a systemic consequence of a para-infectious or post-infectious immune-mediated mechanism $(6,12)$. It is considered that the virus reaches CNS via neuronal retrograde transmission or hematological spread. In addition, the effects on CNS and PNS are thought to occur through the virus entering the cell using ACE-2 receptors $(9,13)$. However, it is not known whether cranial nerve involvement is directly caused by CNS or the direct invasion of peripheral nerves nor is it clear whether this damage is caused directly by the virus or the immune system response triggered by the virus. There are many publications reporting that the carnal nerves are affected by COVID-19. Mao et al. evaluated neurological symptoms in 214 patients infected with COVID-19. It was observed that $36.4 \%$ of these 214 patients who were hospitalized had nervous system findings such as dizziness, headache, taste disturbance, hyposmia, muscle damage, and hemorrhagic and ischemic brain damage (8). However, Mao et al. observed the effects of the virus on peripheral nerves and CNS rather than cranial nerves. We consider that the emergence of neurological findings alone is not an indicator of poor prognosis, as previously assumed because we did not determine a statistically significant difference between the groups with and without cranial nerve involvement in terms of length of hospital stay and intensive care requirement. In particular, both in our study and among the reported cases, the presence of patients with complaints such as sudden vision loss, sudden hearing loss, sudden-onset severe peripheral vertigo, and sudden movement limitation in the eye suggests that COVID-19 involves aggressive neurotropism and neuroinvasion. The current literature contains reports on cranial nerve involvement in patients with COVID-19; e.g., dysphagia caused by N. glossofarengeus, N. vagus and N. hypoglossus damage (12), as well as presence of damage to N. vestibulocochlearis (14), N. facialis (15-17), N. oculomotorius (18, $19)$, N. abducens $(20,21)$, N. trochlearis (22), N. opticus (23) and N. olfactorius (14, 15, 17, 24). COVID-19 has also been reported to cause neurological syndromes such as Guillain-Barré syndrome and Miller Fisher syndrome $(6,20,25)$. Despite all these data, we did not find any study on the prevalence of these cranial nerve symptoms in patients in the current literature. In a study conducted by Mao et al., damage to the peripheral nervous system and CNS was discussed in the majority of patients, but cranial nerve functions were not emphasized, except for taste and smell disorders (8). Bagheri et al. investigated olfactory disorders in people infected with COVID-19, but only used an online survey to identify patients (24). In the current study, we tried to evaluate all cranial nerve involvements together in people infected with COVID-19 and to reveal their prevalence in infected patients. For this purpose, we deemed it appropriate to make a diagnosis based on a direct examination. Only after the patients' discharge from the hospital, we administered the survey through phone calls to determine the development or regression of existing symptoms. However, we can state that there are still some important points that have not yet been fully explained. Although we still cannot explain the exact mechanism, some cranial nerves are affected more frequently in the very early period while others occur later. For example, while patients had symptoms of a very high degree of loss of taste and smell at the time of direct presentation, they rarely presented with ophthalmoparesis, dysphagia, or vision and hearing loss. In a multicentric survey study conducted in 12 hospitals from different regions of Europe, the rate of loss of smell and taste in COVID-19-infected patients was found to be $85.6 \%$ and $88.0 \%$, respectively (26). This led us to consider that the most important factors for this virus settled in the 
respiratory tract to cause cranial nerve damage are the response it triggers in local immunity and direct nerve invasion. As we mentioned earlier, if the most common symptoms of loss of smell (27.2\%) and taste (30.8\%) had occurred as a result of damage caused by the virus in CNS or systemic immune response, we would expect it to occur at a frequency close to other cranial nerve involvement. However, while N. facialis and $\mathrm{N}$. ophthalmicus, the cranial nerves that are closest to the respiratory tract and branch into this region, are frequently affected, N. hypoglossus, N. glossopharyngeus, N. vagus, and N. trigeminus were less frequently involved, which raises further questions that need to be answered. Nevertheless, we believe that the size of the areas in which these nerves innervate in the respiratory tract will naturally increase their exposure, which is a factor to be considered in explaining this situation. Another important finding of our study was that among the patients hospitalized due to COVID-19, those with swallowing disorders due to glossopharyngeal and vagal nerve involvement had a more severe disease process and required a longer hospital stay since their food intake was impaired.

Limitations: The major limiting factor of our study is that we did not have any evidence (such as pathological examination or nerve biopsy sample) to show the direct damage of the virus on the nerves. Although we are not sure, we cannot go beyond predicting whether this damage occurs as a result of the direct effect of the virus or the development of an immune response-mediated mechanism. In addition, there are publications indicating that that the virus is frequently mutated, but we did not know whether the virus type in the patients we evaluated was mutated or whether all patients were infected with the same strain, which can be considered as another limitation.

In conclusion, the COVID-19 disease caused by the SARS-CoV2 virus commonly leads to cranial nerve symptoms. During our study, we observed symptoms of the involvement of very different cranial nerves apart from taste and smell disorders reported in previous studies. However, further studies are needed to provide more definitive results concerning whether these nerve damages are permanent or temporary. Our first impression is that symptoms such as smell, taste, vision and sudden hearing loss, vertigo, swallowing disorders, hoarseness, eye symptoms, and facial hypoesthesia completely disappear within the first month of the infection. Taste and smell disorders rarely last more than one month. Therefore, we consider that these symptoms often do not require any special treatment, and cranial nerve symptoms regress with the current COVID-19 treatment protocol. In addition, as an interesting finding, we determined the dominant effect of cranial nerve involvement on sensory dysfunction compared to motor functions, suggesting that the virus causes more sensory dysfunction. While the negative effects of the virus on sensory functions are evident, motor functions are less frequently affected.

\section{Conflict of Interest}

The authors have no conflicts of interest to declare.

\section{Financial Support}

This research received no specific grant from any funding agency, commercial or not-for-profit sectors.

\section{Ethical Standards}

The authors assert that all procedures contributing to this work comply with the ethical standards of the relevant national and international guides on clinical research. Ethics committee approval was obtained from Harran University Faculty of Medicine 29.06.2020 date and numbered HRU/20.12.09.

\section{Author Contributions:}

All authors cared for the patient and contributed to the manuscript.

\section{References}

1. Sedaghat Z, Karimi N. Guillain Barre syndrome associated with COVID-19 infection: a case report. Journal of Clinical Neuroscience. 2020. 
2. Li G, De Clercq E. Therapeutic options for the 2019 novel coronavirus (2019-nCoV). Nature Publishing Group; 2020.

3. Wang W, Tang J, Wei F. Updated understanding of the outbreak of 2019 novel coronavirus (2019-nCoV) in Wuhan, China. J Med Virol. 2020;92(4):441-7.

4. Chen N, Zhou M, Dong X, Qu J, Gong F, Han Y, et al. Epidemiological and clinical characteristics of 99 cases of 2019 novel coronavirus pneumonia in Wuhan, China: a descriptive study. The Lancet. 2020;395(10223):507-13.

5. Conde G, Pájaro LDQ, Marzola IDQ, Villegas YR, Salazar LRM. Neurotropism of SARS-CoV 2: Mechanisms and manifestations. J Neurol Sci. 2020.

6. Ellul MA, Benjamin L, Singh B, Lant S, Michael BD, Easton A, et al. Neurological associations of COVID-19. Lancet Neurol. 2020;19(9):767-83.

7. Fotuhi M, Mian A, Meysami S, Raji CA. Neurobiology of COVID-19. J Alzheimers Dis. 2020;76(1):3-19.

8. Mao L, Jin H, Wang M, Hu Y, Chen S, He Q, et al. Neurologic Manifestations of Hospitalized Patients With Coronavirus Disease 2019 in Wuhan, China. JAMA Neurol. 2020;77(6):683-90.

9. Yachou Y, El Idrissi A, Belapasov V, Ait Benali S. Neuroinvasion, neurotropic, and neuroinflammatory events of SARS-CoV-2: understanding the neurological manifestations in COVID-19 patients. Neurol Sci. 2020;41(10):2657-69.

10. Yavarpour-Bali H, Ghasemi-Kasman M. Update on neurological manifestations of COVID-19. Life Sci. 2020;257:118063.

11. Poyiadji N, Shahin G, Noujaim D, Stone M, Patel S, Griffith B. COVID-19-associated acute hemorrhagic necrotizing encephalopathy: CT and MRI features. J Radiology. 2020:201187.

12. Cavalagli A, Peiti G, Conti C, Penati R, Vavassori F, Taveggia G. Cranial nerves impairment in post-acute oropharyngeal dysphagia after COVID-19: a case report. Eur j phys rehab med

2020.

13. Chen N, Zhou M, Dong X, Qu J, Gong F, Han Y, et al. Epidemiological and clinical characteristics of 99 cases of 2019 novel coronavirus pneumonia in Wuhan, China: a descriptive study. Lancet. 2020;395(10223):507-13.

14. Chen X, Laurent S, Onur OA, Kleineberg NN, Fink GR, Schweitzer F, et al. A systematic review of neurological symptoms and complications of COVID-19. J Neurol. 2020:1-11.

15. Homma Y, Watanabe M, Inoue K, Moritaka T. Coronavirus Disease-19 Pneumonia with Facial Nerve Palsy and Olfactory Disturbance. Intern Med. 2020;59(14):1773-5.

16. Lima MA, Silva MTT, Soares CN, Coutinho R, Oliveira HS, Afonso L, et al. Peripheral facial nerve palsy associated with COVID-19. J Neurovirol. 2020;26(6):941-4.

17. Xydakis MS, Dehgani-Mobaraki P, Holbrook EH, Geisthoff UW, Bauer C, Hautefort C, et al. Smell and taste dysfunction in patients with COVID-19. Lancet Infect Dis. 2020;20(9):1015-6.

18. Faucher A, Rey PA, Aguadisch E, Degos B. Isolated post SARS-CoV-2 diplopia. J Neurol. 2020;267(11):3128-9.

19. Belghmaidi S, Nassih H, Boutgayout S, El Fakiri K, El Qadiry R, Hajji I, et al. Third Cranial Nerve Palsy Presenting with Unilateral Diplopia and Strabismus in a 24-Year-Old Woman with COVID-19. Am J Case Rep. 2020;21:e925897.

20. Dinkin M, Gao V, Kahan J, Bobker S, Simonetto M, Wechsler P, et al. COVID-19 presenting with ophthalmoparesis from cranial nerve palsy. Neurology. 2020;95(5):221-3. 
21. Falcone MM, Rong AJ, Salazar H, Redick DW, Falcone S, Cavuoto KM. Acute abducens nerve palsy in a patient with the novel coronavirus disease (COVID-19). J AAPOS. 2020;24(4):216-7.

22. Oliveira RMC, Santos DH, Olivetti BC, Takahashi JT. Bilateral trochlear nerve palsy due to cerebral vasculitis related to COVID-19 infection. Arq Neuropsiquiatr. 2020;78(6):385-6.

23. Selvaraj V, Sacchetti D, Finn A, Dapaah-Afriyie K. Acute Vision Loss in a Patient with COVID-19. R I Med J (2013). 2020;103(6):37-8.

24. Bagheri SH, Asghari A, Farhadi M, Shamshiri AR, Kabir A, Kamrava SK, et al. Coincidence of COVID-19 epidemic and olfactory dysfunction outbreak in Iran. Med J Islam Repub Iran. 2020;34:62.

25. Sedaghat Z, Karimi N. Guillain Barre syndrome associated with COVID-19 infection: A case report. J Clin Neurosci. 2020;76:233-5.

26. Lechien JR, Chiesa-Estomba CM, De Siati DR, Horoi M, Le Bon SD, Rodriguez A, et al. Olfactory and gustatory dysfunctions as a clinical presentation of mild-to-moderate forms of the coronavirus disease (COVID-19): a multicenter European study. Eur Arch Otorhinolaryngol. 2020;277(8):2251-61.

Table 1. Frequency distribution of the cases in terms of the localization of cranial nerve involvement

Table 2. Demographic and clinical characteristics of the cases according to the groups with and without cranial nerve involvement

Table 3. Frequency distribution in terms of the complaints of the patients according to the groups with and without cranial nerve involvement

Table 4. Results of the multivariate logistic regression analysis of the data adjusted for age and gender

Figure 1. S urvey form

Figure 2. Rate of the cranial nerve symptoms of the patients according to the diagnostic method

Figure 3 . Olfactory area

Figure 4. Inflammatory cells in the lamina propria and submucosal serous glands in the nasal mucosa tissue lined with a single-row atrophic cubic epithelium (H\&E x 40) 


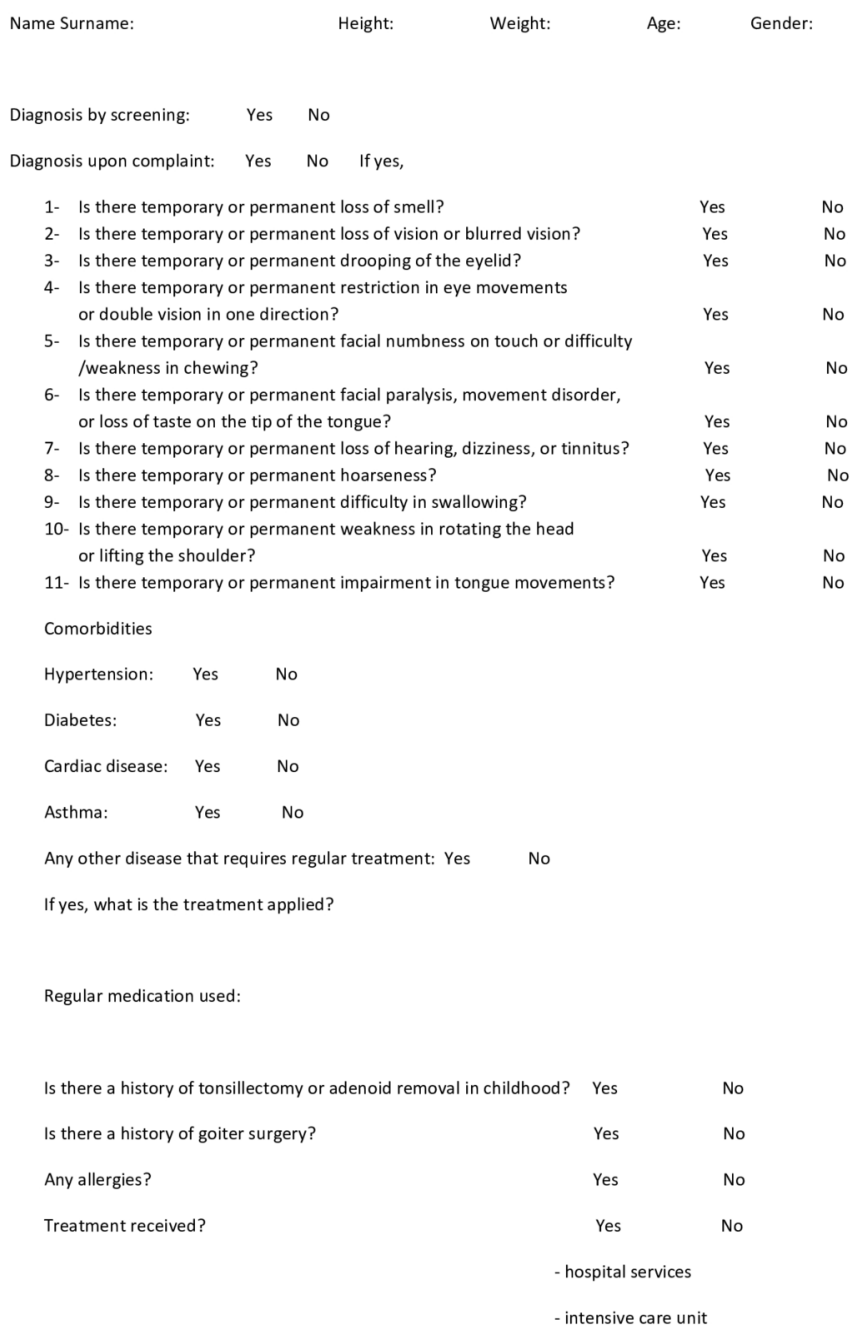



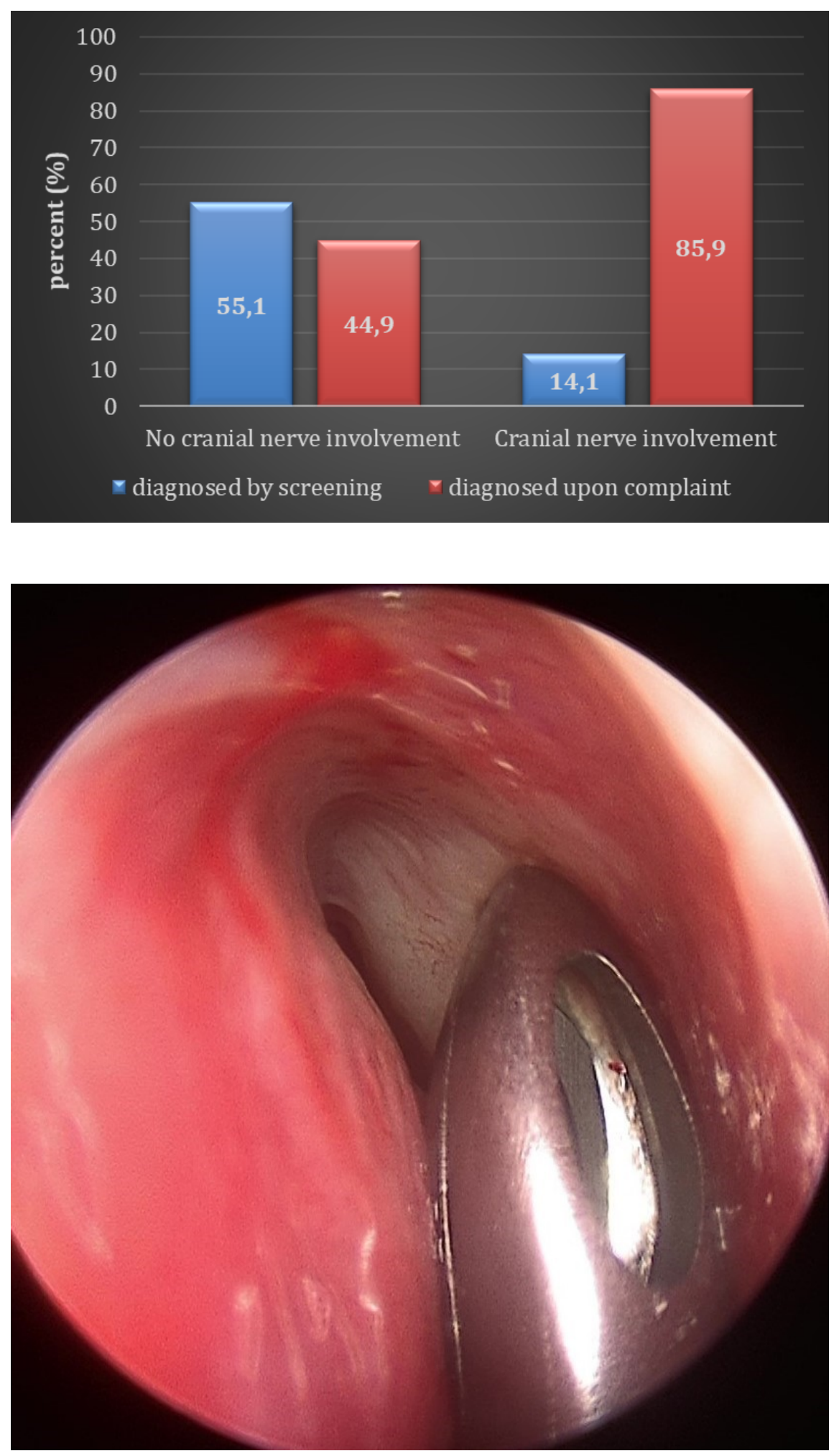


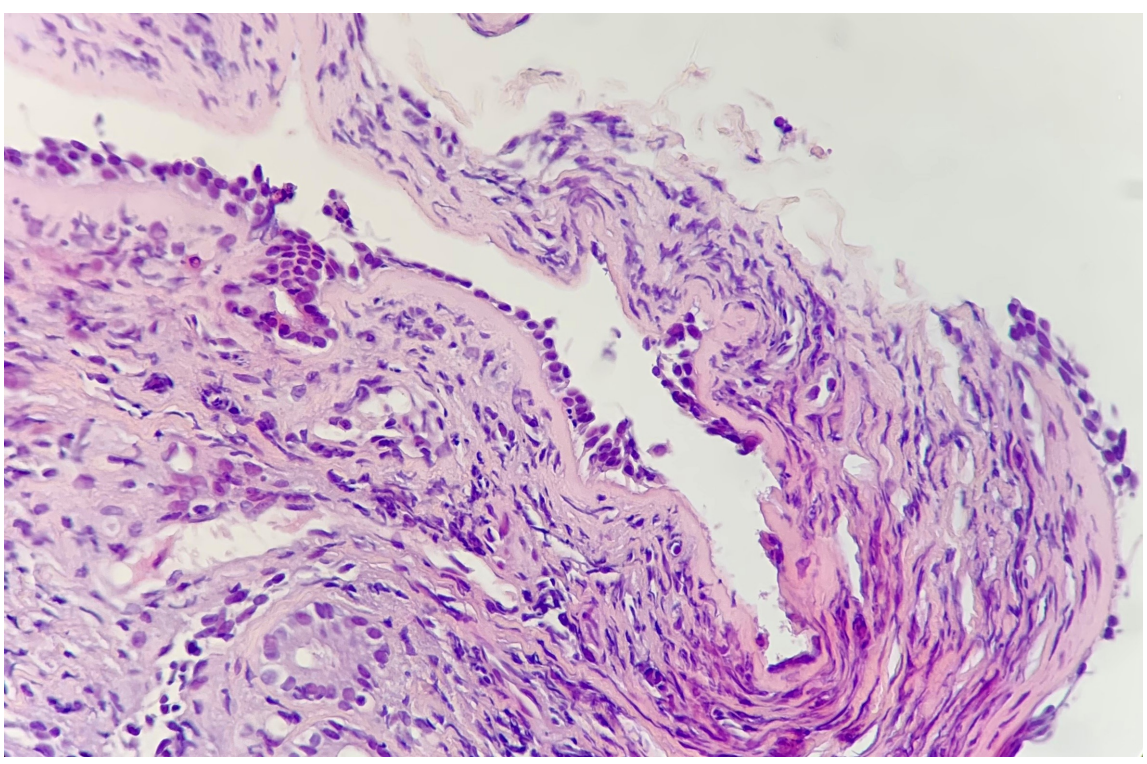

\section{Hosted file}

table 1.pdf available at

https://authorea.com/users/379455/articles/513463-cranial-nerveinvolvement-in-covid-19

\section{Hosted file}

table 2.pdf available at

https://authorea.com/users/379455/articles/513463-cranial-nerveinvolvement-in-covid-19

\section{Hosted file}

table 3.pdf available at involvement-in-covid-19

https://authorea.com/users/379455/articles/513463-cranial-nerve-

\section{Hosted file}

table 4.pdf available at

https://authorea.com/users/379455/articles/513463-cranial-nerve-

involvement-in-covid-19 